\title{
Apoio Matricial nas açôes de Alimentação e Nutrição: visão dos profissionais da Estratégia Saúde da Família
}

I ${ }^{1}$ Ana Lúcia de Magalhães Fittipaldi, 2 Denise Cavalcante de Barros,

${ }^{3}$ Valéria Ferreira Romano I

Resumo: O artigo teve como objetivo apresentar os significados atribuídos pelos profissionais da Estratégia Saúde da Família e do Núcleo de Apoio à Saúde da Família ao Apoio Matricial nas ações de Alimentação e Nutrição como estratégia para propor ações que potencializem a atenção nutricional no território. Trata-se de uma pesquisa qualitativa, em que foram realizadas 18 entrevistas semiestruturadas e analisadas mediante Análise de Conteúdo. As falas dos entrevistados foram classificadas em temas, compondo os eixos norteadores da discussão dos resultados, a saber: ênfase na educação em saúde; ampliação de ações intersetoriais; qualificação das equipes e competências do profissional nutricionista. Os entrevistados ressaltaram a atuação do nutricionista como referência no processo de matriciamento nessa área do conhecimento; consideraram as açôes educativas relevantes no seu cotidiano de trabalho e uma importante estratégia para atender às complexas demandas de alimentação e nutrição; não compreenderam as razões da pouca adesão da população às atividades de educação em saúde e pouco vivenciaram o trabalho interdisciplinar. Como consideraçōes finais, destaca-se que ouvir os envolvidos no processo de trabalho é fundamental para que haja sucesso na implementação e na viabilização do trabalho do Apoio Matricial em Alimentação e Nutrição a partir das necessidades das equipes e comunidades.

> Palavras-chave: pesquisa qualitativa; Estratégia Saúde da Família; nutrição.

\author{
1 Escola Nacional de Saúde \\ Pública Sérgio Arouca, \\ Fundação Oswaldo Cruz. Rio de \\ Janeiro-RJ, Brasil (fittipaldiana@ \\ gmail.com) \\ 2 Escola Nacional de Saúde \\ Pública Sérgio Arouca, \\ Fundação Oswaldo Cruz. Rio de \\ Janeiro-RJ, Brasil (barrosdc@ \\ ensp.fiocruz.br) \\ ${ }^{3}$ Medicina de Família e \\ Comunidade, Universidade \\ Federal do Rio de Janeiro. \\ Rio de Janeiro-RJ, Brasil \\ (valeriaromano34@gmail.com)
}




\section{Introdução}

A Atenção Básica $(\mathrm{AB})$ no Brasil tem sido considerada eixo estruturante do Sistema Único de Saúde (SUS) e, desde a aprovação da Política Nacional de Atenção Básica (PNAB) (BRASIL,2006), está na agenda de prioridades do Ministério da Saúde (MS) como um movimento de ampliação do acesso, melhoria da qualidade e resolubilidade das ações desenvolvidas pelas equipes da Estratégia Saúde da Família (ESF) (BRASIL, 2014a).

Nesse contexto, o MS criou, em 2008, o Núcleo de Apoio à Saúde da Família (NASF), com o objetivo de ampliar o escopo e a resolubilidade das açōes das equipes da ESF, além de atuar no apoio especializado a elas por meio do compartilhamento das responsabilidades e saberes das diversas áreas do conhecimento envolvidas na complexidade do lidar com situações de vida e saúde da comunidade (BRASIL, 2008).

As ações do NASF são orientadas pelo referencial teórico do Apoio Matricial, metodologia de trabalho que propõe a superação das limitações impostas pela organização tradicional dos serviços de saúde, ampliando as possibilidades de integração dialógica entre diversas especialidades do campo da saúde (CUNHA, 2011). Dessa maneira, o Apoio Matricial é uma estratégia que objetiva integrar as equipes da ESF às equipes de NASF por meio do compartilhamento dos problemas do território, da troca de saberes e práticas, da corresponsabilização pelas condutas e intervenções, considerando-se as responsabilidades comuns e específicas de cada núcleo de conhecimento (BRASIL, 2014a). É relevante complementar o conceito de Apoio Matricial com os conceitos de Núcleo e Campo no sentido de contribuir para a compreensão da mudança do processo de trabalho na lógica do compartilhamento dos saberes (CUNHA, 2011). O Núcleo constitui um conjunto de conhecimentos e tarefas específicos, atribuídos a cada especialidade profissional, representando a identidade e a especificidade das diversas disciplinas. O Campo representa um conjunto de tarefas e conhecimentos que uma especialidade deve adquirir para garantir a eficácia e efetividade de suas açóes, representando uma abertura ao mundo da interdisciplinaridade (CUNHA, 2011).

Este artigo aborda a temática da Alimentação e Nutrição, bem como a atuação do profissional nutricionista, entendendo ser este um núcleo do conhecimento que compõe o NASF e que vem se conformando e organizando suas ações prioritárias 
na $\mathrm{AB}$, orientada pelas diretrizes da Política Nacional de Alimentação e Nutrição

(PNAN) (BRASIL, 2012a), sob foco da Organização da Atenção Nutricional. Cabe destacar que a PNAN passou por uma revisão de suas diretrizes com o objetivo de fortalecer sua interface entre o SUS e a PNAB (BRASIL, 2012b).

$A$ inserção do profissional nutricionista na $\mathrm{AB}$ por meio das equipes de NASF tem levado em consideração a situação epidemiológica brasileira atual, definida a partir de uma concomitante tripla carga de doenças: a persistência de doenças infecciosas, desnutrição e problemas de saúde reprodutiva; o desafio das doenças crônicas e de seus fatores de riscos, como tabagismo, sobrepeso, inatividade física, uso excessivo de álcool e outras drogas e alimentação inadequada; e, por último, o crescimento da violência e morte por causas externas.

A situação nutricional que acompanha esse cenário reflete um processo de transição nutricional, com aumento do sobrepeso e obesidade como resultado de mudanças na alimentação, com a crescente oferta de alimentos industrializados, e do acesso a alimentos caloricamente densos e mais baratos e, ainda, da redução da atividade física regular. Essa transição nutricional favorece o incremento das condiçōes crônicas representando um desafio para a atenção nutricional na $\mathrm{AB}$ (MENDES, 2011).

Diante desse cenário, a atuação do profissional de nutrição no NASF tem por objetivo ampliar a resolubilidade das ações de Alimentação e Nutrição prestadas pelas equipes da ESF por meio do Apoio Matricial a elas, nas suas dimensóes técnico-pedagógicas e clínico-assistenciais (BRASIL,2014a).

Para alcançar esse objetivo, algumas estratégias são utilizadas, tendo como norteadoras as diretrizes da PNAN (2012a): promover práticas alimentares saudáveis individuais e coletivas em todos os ciclos de vida; contribuir com respostas às demandas assistenciais nos agravos nutricionais; desenvolver projetos terapêuticos focados nas Doenças Crônicas não Transmissíveis (DCNT); realizar diagnóstico alimentar e nutricional da população, identificando grupos de risco; e promover a Segurança Alimentar e Nutricional (SAN) com vistas a assegurar o Direito Humano à Alimentação Adequada (DHAA) (SILVA et al., 2013).

$\mathrm{Na}$ relação que interliga a atenção nutricional com a $\mathrm{AB}$ (que compreende, entre outras questóes, os cuidados voltados à promoção e proteção da saúde, prevenção, diagnóstico e tratamento de agravos), valorizamos o pensar sobre uma natural interação com as demais ações de atenção à saúde do SUS, fazendo 
supor que, tornando-se um dos elementos da Rede de Atenção à Saúde (RAS), a Nutrição deve prezar pelo princípio da integralidade como norteador de suas ações (SILVA et al., 2013).

No entanto, sobre que conceito de integralidade estamos falando? Certamente sobre um conceito que, como um dos princípios do SUS, prevê três sentidos principais relacionados com: a abordagem integral dos indivíduos, considerando as diferentes dimensões de sua vida; o modo de organizar os serviços, com base na implementação de redes, integrando diversos pontos de atenção para suprir as diversas necessidades dos usuários; e a construção de políticas públicas que garantam acesso a todos os níveis de atenção (BRASIL, 2014a).

As ações de Alimentação e Nutrição na $\mathrm{AB}$ que defendemos articulam todos esses elementos da integralidade, promovendo intersetorialidade e ampliando o olhar para a determinação da saúde, no qual os aspectos psicológicos, sociais, econômicos, ambientais, políticos e culturais convivem com a dimensão biológica, na compreensão sobre o lidar com as questôes nutricionais (SILVA et al., 2013).

Nesse contexto, foi elaborado, no Brasil, o Marco de Referência de Educação Alimentar e Nutricional para as Políticas Públicas, considerando a Educação Alimentar e Nutricional (EAN) como uma importante estratégia para qualificar e promover uma agenda de alimentação e nutrição multidisciplinar e intersetorial, ampliando um campo comum de reflexão sobre a saúde (BRASIL, 2012c).

Sendo a EAN uma estratégia utilizada para a prevenção e controle dos agravos alimentares e nutricionais contemporâneos, ela enfoca os problemas cotidianos da população brasileira, contribuindo, assim, para abordagens relacionadas, por exemplo, com a prevenção e tratamento das DCNT, com as carências nutricionais nos diferentes ciclos de vida, com a valorização e fortalecimento de hábitos regionais, com as diferentes manifestações da cultura alimentar, com a sustentabilidade por meio do controle do desperdício de alimentos e com o incentivo ao consumo de alimentos saudáveis (BRASIL, 2012c).

A EAN, portanto, tem o potencial de dialogar intersetorial e multidisciplinarmente, pressupondo um envolvimento entre as equipes da ESF e do NASF na comunidade.

Assim, a área de Alimentação e Nutrição possui atribuiçõos bem definidas e estabelecidas por meio de políticas públicas, planos de ações e estratégias (BRASIL, 2012a; BRASIL, 2014a; BRASIL, 2011; OMS, 2004), e em um crescente vem se fortalecendo ao longo dos anos. A proposta da PNAB indica que 
essas atribuições devem ser executadas por meio do Apoio Matricial às equipes da ESF, realizado pelos profissionais nutricionistas inseridos nas equipes do NASF (BRASIL, 2012b).

As experiências encontradas na literatura mostram que a atuação do profissional nutricionista no contexto do Apoio Matricial é ainda recente, impregnada pelas distorções comuns na sua formação e na dos profissionais de saúde em geral, necessitando, portanto, ainda ser problematizada. As dificuldades encontradas vão desde o desconhecimento dos pressupostos teóricos e práticos que irão compor a sua agenda de trabalho, passando pelas fragilidades na atuação profissional na lógica do Apoio Matricial, nos sentidos que os profissionais atribuem ao modelo de cuidado, o precário foco no atendimento coletivo, além de problemas na gestão e infraestrutura das Unidades Básicas de Saúde (UBS), falta de apoio institucional, grande demanda do serviço e dificuldade de articular atividades em comum com outros profissionais (FARIAS, 2012; MANCUSO, 2012).

Segundo Silva (2012), o processo de expansão da ESF, concomitante à implantação das equipes de NASF no município do Rio de Janeiro, dificultou a organização do trabalho e a qualificação das equipes. A despeito disso, três questôes foram identificadas como facilitadoras da inserção do nutricionista no NASF, todas estruturais: o perfil nutricional da população brasileira, a insegurança alimentar e o cuidado em saúde abrangendo todas as fases do curso da vida, além de uma tendência da área da saúde à predisposição ao trabalho em equipe para lidar com demandas complexas frequentemente encontradas na $\mathrm{AB}$.

Um estudo realizado com gestores da rede básica de saúde da baixada santista, São Paulo (LAPORTE-PINFILDI, 2016), identificou que a presença insuficiente de profissionais nutricionistas repercutiu negativamente na atenção nutricional no pré-natal e no puerpério.

Outro estudo realizado nas equipes de NASF do estado de Goiás (AGUIAR, 2015) que analisou a formação e a atuação dos nutricionistas identificou que os profissionais possuíam pouca experiência, sentiam-se pouco qualificados para atuar na ESF e no NASF e apresentavam pouca compreensão da realidade socioeconômica, política e cultural do território, resultante da formação acadêmica que não propiciava conhecimento e segurança para atuação na área.

Um estudo de Florindo (2016), em âmbito nacional, demonstrou que a prevalência de ações de alimentação saudável foi maior nos municípios que tinham 
NASF, em comparação com aqueles que não tinham. A presença do profissional nutricionista nas equipes de NASF foi, também, decisiva para que essas ações fossem desenvolvidas com maior frequência. Segundo Vasconcelos (2015), com a expansão da ESF no Brasil, houve também expansão das equipes de NASF e do número de profissionais nutricionistas, sendo estes identificados como o terceiro profissional não médico integrante em termos numéricos, seguidos dos fisioterapeutas e psicólogos. Ainda segundo esse autor, é necessário aprofundar os estudos para avaliar se esta expansão quantitativa vem acompanhada ou não da mudança da lógica da atenção nutricional, priorizando o atendimento compartilhado e interdisciplinar.

Essa expansão da presença do nutricionista nas equipes de NASF, acompanhada pelo investimento na sua formação para a atuação na lógica do Apoio Matricial, fortalece a oportunidade de qualificar a $\mathrm{AB}$ para as açôes de atenção nutricional preconizadas pela PNAN (BRASIL, 2012a).

Diante do exposto, este artigo tem por objetivo identificar os significados que profissionais da ESF com atuação em uma comunidade da zona norte do município do Rio de Janeiro atribuem ao Apoio Matricial nas açôes de Alimentação e Nutrição como estratégia para propor coletivamente ações que potencializem a atenção nutricional no território.

\section{Método}

O presente artigo é fruto de uma dissertação de Mestrado Profissional em Atenção Primária com ênfase na ESF, na Escola Nacional de Saúde Pública Sergio Arouca (ENSP) / Fiocruz.

Foi desenvolvida uma pesquisa qualitativa em uma comunidade da zona norte do município do Rio de Janeiro, nas duas UBS com responsabilidade sanitária pelo território. Os critérios para a escolha do cenário da pesquisa foram baseados no tempo disponível para a realização do estudo, considerando-se o período limitado em cursos de mestrado, e por representar o lócus de atuação profissional dos pesquisadores responsáveis.

As UBS em questão possuíam 7 equipes da ESF cada uma, sendo uma delas Equipe de Consultório na Rua, totalizando 14 equipes responsáveis pelo território do estudo. Ambas as UBS recebiam Apoio Matricial de uma mesma equipe de NASF, composta pelos seguintes profissionais: um psicólogo, um psiquiatra, um 
cardiologista, um ginecologista, um pediatra, um assistente social, um educador

físico e uma equipe de atenção domiciliar, integrada por um fisioterapeuta, um médico generalista e um técnico de enfermagem.

Os critérios para seleção dos participantes do estudo seguiram os preceitos do método da pesquisa qualitativa, sendo os mesmos definidos a partir da representatividade que os conhecimentos, práticas, experiências, comportamentos e atitudes dos profissionais exerciam sobre o objeto do estudo. Dessa forma, consideramos essencial que todas as equipes da ESF, das duas UBS, fossem representadas pelo menos por um profissional, além da inclusão de profissionais do NASF e dos dois gerentes das UBS. Adicionalmente, foram considerados como critérios de inclusão: o tempo de atuação na $\mathrm{AB}$, as diferentes formações acadêmicas, a disponibilidade e o aceite dos profissionais em participar da pesquisa.

Os entrevistados identificados por letras, conforme inserção profissional, e numerados aleatoriamente estão assim discriminados: $(\mathrm{E})$ : profissionais das equipes da ESF; (N): profissionais do NASF e $(\mathrm{G})$ : gerentes das UBS. Para coleta de dados, foi utilizada a entrevista semiestruturada, a qual permite que o entrevistado possa discorrer e expressar livremente seus pontos de vista. Esse tipo de entrevista prevê uma interação entre entrevistado e entrevistador, facilitando as respostas espontâneas, podendo, inclusive, surgir questões inesperadas e de grande valia para o estudo (MINAYO, 2010).

Essa técnica foi realizada a partir de um roteiro predefinido, com os seguintes questionamentos elaborados pelas autoras:

a) Como é o entendimento dos profissionais da ESF, do NASF e dos gerentes sobre o Apoio Matricial nas ações de Alimentação e Nutrição?

b) Como está sendo realizado o Apoio Matricial nas ações de Alimentação e Nutrição nas Unidades de Saúde/Equipes?

c) Quais as demandas e expectativas das equipes para o Apoio Matricial nas ações de Alimentação e Nutrição?

As entrevistas foram realizadas na ENSP, em sala previamente agendada, garantindo a não interrupção e a privacidade dos entrevistados. Foram conduzidas pela pesquisadora responsável, individualmente, no período de janeiro a maio de 2013, e tiveram duração média de 37 minutos e 53 segundos. Foi utilizado o recurso da gravação e realizada posteriormente a transcrição dos depoimentos pela própria entrevistadora. Antes do início da entrevista, foi feita uma apresentação 
da pesquisadora e uma breve descrição da pesquisa, além da leitura e assinaturas do Termo de Consentimento Livre e Esclarecido (TCLE).

Para análise e interpretação dos resultados, foi utilizada a Análise de Conteúdo na sua vertente temática, que infere um tema como conceito central a partir do conteúdo que as entrevistas revelam (MINAYO, 2012; BARDIN, 2011). Esta compreendeu três etapas consecutivas: pré-análise, exploração do material e interpretação dos resultados (BARDIN, 2011). Uma leitura exaustiva do material foi a etapa inicial de pré-análise, com o propósito de obter uma visão geral e identificar as categorias empíricas para classificação das falas. Em seguida, a exploração do material representou a análise em si, permitindo a classificação das falas, ou de fragmentos selecionados delas, entre as categorias de análise. Em sequência, foram identificados núcleos de sentido em cada categoria empírica que, juntamente com os pressupostos teóricos iniciais, apontaram os temas em torno dos quais foi realizada a interpretação dos depoimentos. Os temas em questão direcionaram a organização da apresentação dos resultados e da discussão.

A pesquisa foi aprovada pelo Comitê de Ética em Pesquisa (CEP) da ENSP/ Fiocruz, sob Parecer no 178.224, em 19 de dezembro de 2012, e cumpre as disposições da Resolução no 466/2012, referente a pesquisas envolvendo seres humanos (BRASIL, 2012d).

\section{Resultados e Discussão}

Foram realizadas 18 entrevistas na pesquisa de campo com um representante de cada equipe da ESF, dois profissionais do NASF e dois gerentes das unidades de saúde, com as seguintes formações acadêmicas: nove enfermeiros, oito médicos e um psicólogo.

Os temas empíricos identificados na análise das entrevistas foram: ênfase na educação em saúde; ampliação de açōes intersetoriais; qualificação das equipes para as ações de alimentação e nutrição; e competências do profissional nutricionista.

\section{Ênfase na educação em saúde}

Os entrevistados identificaram que as atividades educativas coletivas são potencialmente importantes na área de Alimentação e Nutrição, na medida em que atingem um maior número de pessoas, permitindo troca de experiências mais amplas. 
Acho que em grupo mesmo. Educação pra população. Pegar em massa. Porque se for individual não vai dar conta. A demanda é grande. Então, e, fazendo grupos educativos atenderia muito mais. Sem sombra de dúvidas. E é uma forma deles passarem as experiências. Porque cada um, cada família tem uma vivência, uma experiência, acredita em alguma coisa. [...]. Então essa troca de informações e saberes eu acho bem bacana. E aí o profissional de nutrição que vai saber tranquilamente conduzir aquilo da melhor maneira possível (E7).

Destacaram ainda que as ações educativas deveriam estar voltadas para todos os usuários, porém com destaque para a prevenção e tratamento das DCNT, encontradas frequentemente nos usuários da $\mathrm{AB}$.

Eu acho que seria interessante às vezes até trabalhar em conjunto, em grupo de hipertensos e diabéticos, né? A gente fazer um grupo não só com a parte médica, mas um grupo multidisciplinar é, da nutrição, das outras áreas, pra gente poder lidar um pouco melhor com isso, passar informações, né? Melhores e com a qualidade maior pra esses grupos e com isso acabar melhorando essa questão da alimentação, da nutrição [...] (E13).

Uma das diretrizes do NASF é a Educação Popular em Saúde, envolvendo a população na construção participativa do seu próprio cuidado de saúde (OLIVEIRA, 2010). Segundo Freire (1996), a educação é entendida como uma prática pedagógica participativa, a qual precisa acolher as condições objetivas dos sujeitos (que o fazem viver de determinado modo) e suas representações subjetivas (que o fazem interpretar o seu lugar no mundo).

Para além de provocar mudanças de hábitos na população com o objetivo de prevenir um conjunto de doenças, a Educação em Saúde deve ser entendida como uma estratégia de compreender os sentidos e as causas das doenças, como preveni-las e superá-las (GOMES, 2011). Assim, a Educação em Saúde, nos moldes da concepção de Paulo Freire sobre processos educativos, considera os saberes prévios dos educandos, enfatiza a não transmissão de conhecimentos, buscando, enfim, uma relação de troca entre o saber popular e o científico, capaz de construir de forma compartilhada a solução dos problemas que incomodam e oprimem (FREIRE, 1996).

A Educação em Saúde, portanto, não deve ser entendida como mais uma oferta pontual de serviços, e sim como uma atividade que reorienta as práticas de saúde realizadas, sendo um instrumento de construção da participação popular no cotidiano dos serviços de saúde. Possui, assim, um potencial transformador (GOMES,2011). 

uma grande demanda da população, fruto da mudança do perfil epidemiológico que é determinado pelo processo de envelhecimento populacional com aumento das doenças crônicas em detrimento das doenças infectocontagiosas (MENDES, 2011), conforme abordado acima.

Sabe-se que uma das prioridades da agenda de ações intersetoriais do governo federal tem sido o Plano de combate às DCNT, com ênfase para a prevenção e tratamento do sobrepeso e da obesidade. Assim, as demandas para as ações de Alimentação e Nutrição têm sido crescentes, considerando que as causas da DCNT são multifatoriais e que os fatores de risco identificados estão relacionados, principalmente, com tabagismo, sobrepeso e obesidade, inatividade física e alimentação inadequada (MENDES, 2011; BRASIL, 2011).

Apesar da relevância das atividades educativas, foi pontuada, nas entrevistas, a dificuldade experimentada pelos usuários na adesão às ações coletivas. Essa dificuldade, muitas vezes relatada como resistência ou desaprovação, originase, possivelmente, na cultura curativa dos sistemas de saúde. A prevenção, na formação em saúde e no modelo tecnoassistencial usual, tem estado relegada a segundo plano, mantendo uma prática na qual as unidades de saúde têm sido procuradas quase exclusivamente para tratamento individualizado de doenças.

Acho que trabalhar em grupo seria muito legal. Hoje a gente tem muita dificuldade pra trabalhar em grupo. Dificuldade da própria equipe e dificuldade dos próprios pacientes aderirem ao grupo. Mas quando você trabalha em grupo, quando você tem informações de outras pessoas, aderem mais, conhecem mais, eu acho que trabalhar em grupo seria muito interessante Apesar dos pacientes não gostarem muito (E6).

Segundo Merhy (2013), e focando nossa discussão nas dificuldades dos profissionais de saúde em prover cuidado, sublinhamos que se eles não considerarem os saberes que cada usuário tem de si, do mundo e dos outros, possíveis fracassos terapêuticos se perpetuarão. As disputas entre o autocuidado e o prescrito, o pensamento científico e o pensamento popular, revelam dissonâncias e ambiguidades na forma de cuidar e na concepção sobre saúde. Aprender a ouvir os diversos saberes, sem negá-los ou desvalorizá-los, parece ser o mais razoável a ser feito, se o que consideramos importante for a produção da vida, e não da doença.

O Marco de Referência de EAN para as políticas públicas (BRASIL, 2012c) recomenda que abordagens educativas devam incorporar os conhecimentos populares contextualizados nas realidades dos sujeitos, famílias e comunidades, 
promovendo a sua autonomia e liberdade para realizar suas escolhas alimentares

perante diversas opções de consumo que são apresentadas cotidianamente. Por isso, reforçamos que as práticas de educação em saúde devem ser pensadas para além da transmissão de conhecimentos, desenvolvendo senso crítico sobre situações do dia a dia, buscando soluções e alternativas possíveis.

Provocamos, assim, uma reflexão sobre a baixa adesão às atividades educativas na $\mathrm{AB}$. Talvez elas não estejam sendo planejadas incluindo a participação do próprio usuário. Será que os profissionais de saúde aprenderam a ouvir as reais necessidades da comunidade? Vejamos o seguinte relato:

É, a gente fica fechado fazendo o consultório, com o especialista, com o apoiador do NASF, mas na realidade não é nada daquilo, ninguém foi na comunidade pra ver como é a reutilização de alimentos, se pode potencializar. É pensar no grupo, não pensar isoladamente (G1).

Sobre o planejamento das ações educativas, é preciso que seja realizado de forma participativa, para que os usuários se sintam envolvidos nos processos decisórios e possam se comprometer com a efetividade e sustentabilidade das ações. Assim, profissionais e usuários, planejando juntos, poderão identificar as suas reais necessidades mútuas (BRASIL, 2012c).

\section{Ampliação de ações intersetoriais}

Fica cada vez mais claro que as ações de Alimentação e Nutrição, assim como as demais ações de saúde, necessitam extrapolar o seu olhar para além do estritamente biológico e causal, se seus objetivos forem focados na integralidade.

Dessa maneira, as narrativas ressaltaram a intersetorialidade como fundamental nas ações de Alimentação e Nutrição dentro dos preceitos da Promoção da Saúde, conforme mencionado abaixo:

A gente precisa tá vendo mais a família, de prevenir mais. A gente não tem que ver só hipertenso e diabético, descompensados, emagrecer, pra isso tem a ESF. De estar trabalhando na creche, ver a alimentação na escola, se as crianças já estão começando a ficar obesas. E não só depois que ela necessita de nutricionista e sim antes, a parte preventiva. Prevenir as complicaçóes, a obesidade. E tem como fazer isso (E4).

A intersetorialidade é aqui entendida como a articulação de diversos setores governamentais e da sociedade que leve a uma corresponsabilização pela garantia da Segurança Alimentar e Nutricional da população. Se cada setor ampliar seu olhar como resultado da convivência com a perspectiva dos demais setores envolvidos, certamente soluçōes criativas e inovadoras poderão surgir (BRASIL, 2012a). 

de saúde devem estar organizados para oferecer não só os cuidados curativos e preventivos, mas também ampliar suas ações de forma abrangente e intersetorial. Dessa forma, a área da saúde, ao extrapolar suas fronteiras, envolveria setores sociais, políticos, econômicos e ambientais (BRASIL, 2012a; BRASIL, 2014b), estimulando ações inovadoras e socialmente contributivas ao desenvolvimento dos indivíduos e comunidade. Todos esperados para a consolidação do SUS.

\section{Qualificação das equipes para as ações de Alimentação e Nutrição}

A abordagem técnico-pedagógica do NASF foi identificada como relevante na área de Alimentação e Nutrição.

Os profissionais ressaltaram a importância da presença de um nutricionista na qualificação da equipe, no sentido de ampliar seus conhecimentos na área de Alimentação e Nutrição, por meio da educação permanente, aqui relatada como "treinamento"

Eu acho muito interessante a questão do treinamento. [...] seria interessante dentro do turno da reunião de equipe, por exemplo. Então a gente determinaria, sei lá, hoje a gente vai ter um treinamento com a nutrição. A gente podia fixar isso, entendeu? [...] não fariam uma orientação pros usuários, mas pra equipe como um todo, entendeu? Uma informação única que todo mundo ia falar a mesma língua com o usuário (E8).

A educação permanente em saúde é uma importante diretriz do NASF, pois incorpora o trabalho cotidiano das equipes de saúde no processo de ensinoaprendizagem, configurando o processo de trabalho como fonte do conhecimento e transformando as práticas profissionais em espaços educativos ampliados (BRASIL, 2009).

Nesse contexto de qualificação dos profissionais, vemos a necessidade de definir as possibilidades de atuação do nutricionista como matriciador das equipes da ESF. Identificamos uma oportunidade ímpar de potencializar as ações de Alimentação e Nutrição desenvolvidas pela ESF no seu território de responsabilidade sanitária.

O profissional nutricionista deve desempenhar o papel de facilitador desse processo, sensibilizando e estimulando as equipes a desenvolver um olhar vigilante e crítico sobre as questôes alimentares e nutricionais da população. A sua contribuição deve priorizar a construção de uma agenda integrada para organizar a atenção nutricional a partir das necessidades das equipes e comunidades, com 
base nas diretrizes da PNAN (BRASIL, 2012a), trabalhando assim com as

diversas possibilidades de solução para os problemas nutricionais encontrados na AB. Todavia, essa ainda não parece ser a realidade encontrada no NASF. Caminhos a serem trilhados.

\section{Competências do profissional nutricionista}

As falas parecem ter mostrado que alguns profissionais da saúde ainda preferem que o próprio profissional nutricionista execute as orientações de Alimentação e Nutrição, não incorporando a interdisciplinaridade no seu cotidiano, principalmente nas práticas clínicas.

Mas eu ainda prefiro que isso fique com a nutrição mesmo. O nutricionista orien-
tando, informando. Por que aí é mais uma tarefa para a equipe. Mas se a gente tem o
profissional aqui, e esse acesso é relativamente é tranquilo, é fácil, por que não acionar
o profissional? Fica muito mais completo. A orientação fica muito melhor (E7).

Sobre a interdisciplinaridade, observamos que há uma linha tênue que separa o que é próprio de cada núcleo do conhecimento profissional daquilo que poderia ser compartilhado entre todos. Ou seja, o que é competência exclusiva do núcleo de especialidade e o que pode e deve ser compartilhado no cotidiano das práticas em saúde. Não encontramos respostas, mas apenas pistas.

$\mathrm{O}$ trabalho em equipe exige um processo contínuo de construção, sendo fundamental refletir sobre as especialidades e suas especificidades. A profissão do nutricionista foi regulamentada pela Lei 8.234/1991, a qual estabelece, como atividade privativa do nutricionista, "assistência e educação nutricional a coletividades ou indivíduos, sadios ou enfermos, em instituições públicas e privadas e em consultório de nutrição e dietética" (BRASIL, 1991).

Alguns documentos, como a "Estratégia Global sobre Alimentação, Atividade Física e Saúde" (OMS, 2004), definem como sendo responsabilidade das equipes de saúde a execução das ações de educação nutricional da população, mas pontuando que o profissional nutricionista seja a referência técnica para os demais profissionais envolvidos nas ações. Dessa forma, sugerem que as açôes de promoção da alimentação saudável na $\mathrm{AB}$ não sejam responsabilidade exclusiva do profissional nutricionista, reconhecendo-o, no entanto, como primordial.

Alguns critérios para atendimentos específicos, de competência do nutricionista, foram descritos para casos que ultrapassam a capacidade resolutiva da ESF. Esses casos seriam atendidos pelo mesmo profissional responsável pelo 
Apoio Matricial, evitando encaminhamentos e diluição das responsabilidades. São exemplos desses critérios: doenças crônicas com restrições alimentares; gestantes com diabetes, hipertensão ou excesso de peso; obesidade grave e anorexia nervosa (SARAIVA, 2012).

O MS, por intermédio do Departamento de Atenção Básica, produz e publica diversos materiais técnicos que visam orientar e estimular os profissionais de saúde envolvidos com as ações de incentivo à alimentação saudável. Esses materiais procuram, por meio de linguagem clara e objetiva, ser um guia a ser utilizado e adaptado às realidades locais. O Guia Alimentar para a População Brasileira é um deles (BRASIL, 2014c).

Ao refletir sobre esse guia, verificamos que as orientações divulgadas são destinadas a orientar a população a prevenir doenças e promover a saúde, sendo indicadas à população saudável. As orientaçōes não são dirigidas a grupos vulneráveis ou pessoas com alguma doença já instalada, que implique distúrbio nutricional. Nesse caso, as orientações do Guia Alimentar devem ser adaptadas pelo profissional nutricionista (BRASIL, 2014c).

Percebemos, nas entrelinhas dos discursos, uma preocupação dos profissionais com as questôes de nutrição, indicando como oportuno o papel do nutricionista no estímulo ao uso de materiais técnicos pedagógicos, como o guia alimentar, trabalhando em conjunto com as equipes as diversas possibilidades de campo de práticas para as ações de EAN. A utilização desses materiais pode ser um aliado na tentativa de driblar a falta de tempo em relação à quantidade de atribuiçôes que as equipes possuem e de facilitar o seu processo de trabalho.

Algumas limitações foram encontradas durante a realização do trabalho, como o tempo para a sua execução, o que levou a delimitar o cenário do estudo resultando na descrição de uma realidade local e das especificidades do território em questão.

\section{Considerações finais}

O presente artigo objetivou apresentar os significados que os profissionais que atuam na ESF em uma comunidade do município do Rio de Janeiro atribuem ao Apoio Matricial na área de Alimentação e Nutrição e a sua inserção no cotidiano de trabalho das equipes.

Por meio do estudo realizado e aqui discutido, podemos perceber que os profissionais da $\mathrm{AB}$ identificam diversas dimensões e possibilidades de 
desenvolvimento de ações de alimentação e nutrição, bem como reconhecem a amplitude de atuação do profissional nutricionista no contexto do Apoio Matricial, no âmbito da $\mathrm{AB}$, embora tenham nestas impressões mais prospecçôes do que vivências conjuntas. Parte-se da premissa de que, para que haja sucesso na implantação e na implementação de uma política pública, é fundamental que os profissionais envolvidos sejam ouvidos e possam opinar a respeito.

Observamos que as expectativas que os profissionais manifestaram para o Apoio Matricial nas ações de Alimentação e Nutrição foram direcionadas para as ações educativas coletivas, para o incentivo à intersetorialidade e para a qualificação das equipes da ESF com enfoque nas DCNT. Além disso, pontuaram a atuação do profissional nutricionista como referência no processo de matriciamento nessa área do conhecimento.

A pesquisa revelou que os profissionais consideram as ações educativas relevantes no seu cotidiano de trabalho, entendendo que a troca de experiências e de conhecimento no coletivo seria uma forma efetiva de compreender as diversas dimensões da produção social da saúde. A partir das falas dos entrevistados, emerge uma importante reflexão sobre como o profissional nutricionista pode contribuir com as equipes das ESF.

$\mathrm{Na} \mathrm{PNAB}$, está previsto que as atribuições pertinentes à área de Alimentação e Nutrição sejam executadas por meio do Apoio Matricial às equipes da $A B$, sendo este um caminho para formar uma rede forte de apoio para a implementação de ações efetivas de promoção da saúde que tenham repercussões na saúde da população. Dessa forma, compete ao profissional nutricionista pensar em conjunto com as equipes as estratégias para efetivar as responsabilidades principais do Apoio Matricial: a vertente assistencial (cuidado prestado à população) e a técnico-pedagógica (voltada para a qualificação dos profissionais das equipes).

Nesse contexto, o nutricionista pode contribuir para a organização da AB com enfoque na oferta de ações de cuidado, prevenção e promoção da saúde visando prestar atenção integral à saúde da população a partir de um diagnóstico local. Este diagnóstico propiciará acompanhar o perfil e a evolução nutricional dos indivíduos e coletividades; valorizar os aspectos sociais, econômicos, familiares e culturais da alimentação; ampliar as ações de Alimentação e Nutrição no território; organizar a educação permanente das equipes em vários espaços e propor uma agenda integrada e articulada entre os diversos atores ampliando as possibilidades de ações intersetoriais. 
O presente estudo contribui com elementos importantes para o desenvolvimento do trabalho das equipes da ESF e do NASF, bem como para a consolidação da proposta de trabalho do Apoio Matricial nas ações de Alimentação e Nutrição na AB. Sugere-se, que estudos com maior abrangência sejam realizados, contribuindo para a consolidação da PNAN, em consonância com a PNAB. ${ }^{1}$

\section{Referências}

AGUIAR, C.B.; COSTA, N.M.S.C. Formação e atuação de nutricionistas dos Núcleos de Apoio à Saúde da Família. Rev. Nutr., v. 28, n. 2, p. 207-216, mar-abr/2015.

BARDIN, L. Análise de conteúdo. São Paulo: Edições 70, 2011.

BRASIL. Ministério da Saúde. Secretaria de Atenção à Saúde. Departamento de Atenção Básica. Núcleo de Apoio à Saúde da Família. Cadernos de Atenção Básica, n. 39, Brasília: Ministério da Saúde, 2014a

. Conselho Nacional de Saúde. Resolução nº. 466, de 12 de dezembro de 2012d. Aprova diretrizes e normas regulamentadoras de pesquisas envolvendo seres humanos. Diário Oficial [da] República Federativa do Brasil, Brasília, DF, 13 jun. 2013. Disponível em: <http:// conselho.saude.gov.br/resolucoes/2012/Reso466.pdf>. Acesso em 03 mar. 2016.

. Ministério da Saúde. Portaria no. 154, de 24 de janeiro de 2008. Cria os Núcleos de Apoio à Saúde da Família (NASF). Diário Oficial [da] República Federativa do Brasil, Brasília, DF, 4 mar. 2008.

- Ministério da Saúde. Secretaria de Atenção à Saúde. Departamento de Atenção Básica. Política Nacional de Alimentação e Nutrição. Brasília: Ministério da Saúde, 2012a.

- Ministério da Saúde. Secretaria de Atenção à Saúde. Departamento de Atenção Básica. Política Nacional de Atenção Básica. Brasília: Ministério da Saúde, 2012b.

- Ministério da Saúde. Secretaria de Atenção à Saúde. Departamento de Atenção Básica. Guia alimentar para a População Brasileira. 2. ed. Brasília: Ministério da Saúde, 2014c.

- Ministério da Saúde. Secretaria de Gestão do Trabalho e da Educação na Saúde.

Departamento de Gestão da Educação em Saúde. Política Nacional de Educação Permanente em Saúde. Brasília: Ministério da Saúde, 2009.

- Ministério da Saúde. Secretaria de Vigilância à Saúde. Política Nacional de Promoção da Saúde: PNaPS: revisão da Portaria MS/GM no 687, de 30 de março de 2006. - Brasília: Ministério da Saúde, 2014b. 32 p.

- Ministério da Saúde. Secretaria de Vigilância em Saúde. Departamento de Análise de Situação de Saúde. Plano de açôes estratégicas para o enfrentamento das doenças crônicas não transmissiveis (DCNT) no Brasil 2011-2022. Brasília: Ministério da Saúde, 2011. 
. Ministério do Desenvolvimento Social e Combate à Fome. Secretaria Nacional

de Segurança Alimentar e Nutricional. Marco de referência de educação alimentar e nutricional para as políticas públicas. Brasília: Ministério do Desenvolvimento Social e Combate à Fome, 2012c.

. Lei no ${ }^{\circ}$. 234, de 17 de setembro de 1991. Regulamenta a profissão de nutricionista e dá outras providências. Diário Oficial [da] República Federativa do Brasil, Brasília, DF, 18 set. 1991. Disponível em: <http://www.cfn.org.br/novosite/conteudo.aspx?IDMenu=56>. Acesso em: 02 jul. 2012.

CUNHA, G.T.; CAMPOS G.W.S. Apoio Matricial e Atenção Primária em Saúde. Saúde Soc., São Paulo, v. 20, n. 4, p. 961-70, dez. 2011.

FARIAS, B.P. Atuação do nutricionista em equipe multiprofissional na atenção básica de saúde. 2012. Dissertação (Mestrado em Saúde Pública) - Faculdade de Saúde Pública, São Paulo, 2012.

FLORINDO, A.A. et al. Promoção da atividade física e da alimentação saudável e a saúde da família em municípios com academia da saúde. Rev. bras. educ. fís. esporte, São Paulo, v. 30, n. 4, p. 913-924, dez. 2016.

FREIRE, P. Pedagogia da Autonomia: saberes necessários à prática educativa. São Paulo: Paz e Terra, 1996.

GOMES, L.B.; MERHY, E.E. Compreendendo a educação popular em saúde: um estudo na literatura brasileira. Cad Saude Publica, Rio de Janeiro, v. 27, n. 1, p. 7-18, jan. 2011.

LAPORTE-PINFILDI, A. C. et al. Atenção nutricional no pré-natal e no puerpério: percepção dos gestores da Atenção Básica à Saúde. Rev. nutr.; v.29 n.1: 109-123, jan-fev 2016. MANCUSO, A.M.C. et al. A atuação do nutricionista na Atenção Básica à Saúde em um grande centro urbano. Cien Saude Colet, Rio de Janeiro, v. 17, n. 12, p. 3289-3300, dez. 2012. MENDES, E. V. As redes de atenção à saúde. Brasília: Organização PanAmericana da Saúde, 2011.

MERHY, E.E. Ver a si no ato de cuidar: Educação Permanente na Saúde. In: FERLA, A.A. et al. (Org.). VER-SUS Brasil: Vivências e Estágios na Realidade do Sistema Único de Saúde. Associação Brasileira da Rede Unida; Porto Alegre: Rede Unida, 2013. p.58-71. Disponível em: <http://www.otics.org.br/otics/estacoes-de-observacao/versus/acervo/caderno-detextosdo-ver-sus-brasil/caderno-de-textos-do-ver-sus-brasil-documento-eletronico/view >. Acesso em: 22 ago. 2013.

MINAYO, C.S. (Org). Pesquisa Social: teoria, método e criatividade. 31. ed. Petrópolis: Vozes, 2012.

MINAYO, M.C.S. O desafio do conhecimento: pesquisa qualitativa em saúde. 12. ed. São Paulo: Hucitec, 2010. 
OLIVEIRA, G.N. O Apoio Matricial como Tecnologia de Gestão e Articulação em Rede. In: CAMPOS, G.W.S.; GUERRERO, A.V.P. (Org.). Manual de práticas de atenção básica: saúde ampliada e compartilhada. 2. ed. São Paulo: Hucitec, 2010. p. 273-82.

ORGANIZAÇĀO MUNDIAL DE LA SALUD. 57a Assembléia Mundial de la Salud. Estratégia mundial sobre régimen alimentario, actividad física y salud. Ginebra: Organización Mundial de la Salud, 2004.

SARAIVA, A.S.; ZEPEDA, J. Princípios do Apoio Matricial. In: GUSSO, G.; LOPES, J.M. (Org.). Tratado de Medicina de Família e Comunidade v.1. Porto Alegre: Artmed, 2012. p. 298-306.

SILVA, J.P. A Inserção do nutricionista no núcleo de apoio a saúde da família no município do Rio de Janeiro. 2012. Dissertação (Mestrado em Saúde Pública) - Escola Nacional de Saúde Pública Sérgio Arouca - FIOCRUZ, Rio de Janeiro, 2012.

SILVA, J.P.; BAIÃO, M.R.; SANTOS, M.S. Ações de Alimentação e Nutrição na Atenção Básica. In: BARROS, D.C.; SILVA, D.O.; SANTOS, M.M.A.S.; BAIÃO, M.R. (Org.). Alimentação e Nutrição: fundamentos para a prática em saúde coletiva. Rio de Janeiro: EAD/ENSP, 2013. p. 59-105.

VASCONCELOS, I.A.L.; SOUSA, M.F.de; SANTOS, L.M.P. Evolução do quantitativo de nutricionistas na Atenção Básica do Brasil: a contribuição dos Núcleos de Apoio à Saúde da Família e da Estratégia Saúde da Família de 2007 a 2013. Rev. Nutr., Campinas, v. 28, n. 4, p. 431-450, Aug. 2015.

\section{Nota}

${ }^{1}$ A.L.M. Fittipaldi realizou as entrevistas, a análise e interpretação dos resultados, e redigiu o artigo. D.C de Barros e V.F. Romano realizaram a análise e interpretação dos resultados, e redigiram o artigo. 


\section{Matrix Support in Food and Nutrition actions: the perspective of professionals of the Family Health Strategy}

This article aimed to present the meanings attributed to the professionals of the Family Health Strategy and the Support Center for Family Health in Matrix Support in the actions of Food and Nutrition as a strategy for granting actions that enhance a nutritional care in the territory. It is a qualitative research, in which 18 semistructured interviews were carried out and analyzed through Content Analysis. The interviewees' statements were classified in topics, composing the guiding axes of the discussion of results, that is: emphasis on health education; expansion of intersectoral actions; qualification of the teams and competencies of the nutritionist. The interviewees emphasize the performance of the nutritionist as a reference in the registration process in this area of knowledge; They considered it as educational actions relevant to their daily work and an important strategy to meet the complex demands of food and nutrition. There is nothing like the reasons for the population's low adherence to health education activities and little experience of interdisciplinary work. As final considerations, it is important to note that it involves a fundamental work process in order to have success in the implementation and a viability of the Matrix Support in Food and Nutrition work based on the needs of the teams and communities.

> Keywords: qualitative research; Family Health Strategy; Nutrition. 